\title{
Transitive Lie Algebras of Vector Fields: An Overview
}

\author{
Jan Draisma
}

Received: 15 May 2011 / Accepted: 30 November 2011 / Published online: 9 December 2011 C The Author(s) 2011. This article is published with open access at Springerlink.com

\begin{abstract}
This overview paper is intended as a quick introduction to Lie algebras of vector fields. Originally introduced in the late nineteenth century by Sophus Lie to capture symmetries of ordinary differential equations, these algebras, or infinitesimal groups, are a recurring theme in twentieth-century research on Lie algebras. I will focus on so-called transitive or even primitive Lie algebras, and explain their theory due to Lie, Morozov, Dynkin, Guillemin, Sternberg, Blattner, and others. This paper gives just one, subjective overview of the subject, without trying to be exhaustive.
\end{abstract}

\section{Formal Power Series, Vector Fields, and Lie Algebras}

In this section we introduce the basic objects of study: Lie algebras of formal vector fields. The section concludes with a reading guide to the remaining sections.

Throughout this paper, $K$ will denote a field of characteristic zero, which we will specialise to $\mathbb{R}$ or $\mathbb{C}$ in some convergence questions. Fix a natural number $n$, and let $K[[\mathbf{x}]]:=K\left[\left[x_{1}, \ldots, x_{n}\right]\right]$ denote the $K$-algebra of formal power series in the variables $x_{1}, \ldots, x_{n}$, i.e., series of the form $\sum_{\alpha \in \mathbb{N}^{n}} c_{\alpha} \mathbf{x}^{\alpha}$, where $\mathbf{x}^{\alpha}$ denotes the monomial $x_{1}^{\alpha(1)} \cdots x_{n}^{\alpha(n)}$ and where there are no restrictions on the coefficients $c_{\alpha} \in K$. Every such series is of the form $f=\sum_{d=0}^{\infty} f_{d}$ with $f_{d}$ a homogeneous polynomial of degree $d$ in the variables $x_{1}, \ldots, x_{n}$. The minimal $d$ for which $f_{d}$ is nonzero is called

The author is supported by a Vidi grant from the Netherlands Organisation for Scientific Research (NWO).

\footnotetext{
J. Draisma $(\varangle)$

Department of Mathematics and Computer Science, Technische Universiteit Eindhoven,

P. O. Box 513, 5600 MB Eindhoven, The Netherlands

e-mail: j.draisma@tue.nl

J. Draisma

Centrum voor Wiskunde en Informatica, Amsterdam, The Netherlands
}

Birkhäuser 
the order ord $f$ of $f$. Let $M$ denote the subspace of $K[[\mathbf{x}]]$ of elements of (strictly) positive order. If ord $f>0$, then $f$ can be written (in a non-unique way) as $\sum_{i=1}^{n} x_{i} g_{i}$ where the minimum of the orders of the $g_{i}$ equals ord $f-1$. Iterating this argument (with the $g_{i}$ instead of $f$ in the next iteration) shows that if ord $f>d \geq 0$, then $f$ is of the form $\mathbf{x}^{\alpha_{1}} h_{1}+\cdots+\mathbf{x}^{\alpha_{N}} h_{N}$ with monomials $\mathbf{x}^{\alpha_{i}}, \alpha_{i} \in \mathbb{N}^{n}$ of degree $d$ and all $h_{i}$ of order at least 1. In particular, $f$ is then an element of $M^{d+1}$, the space of (finite) linear combinations of products of $d+1$ elements of $M$. This shows that in the metric on $K[[\mathbf{x}]]$ defined by $d(f, g)=2^{-\operatorname{ord}(f-g)}$ the closed ball around 0 with radius $2^{-(d+1)}$ is contained in $M^{d+1}$; the opposite inclusion is even easier.

By a formal vector field (in $n$ variables) over $K$ we mean a derivation $X$ of the (commutative) formal power series ring $K\left[\left[x_{1}, \ldots, x_{n}\right]\right]=: K[[\mathbf{x}]]$, that is, a $K$-linear map $K[[\mathbf{x}]] \rightarrow K[[\mathbf{x}]]$ satisfying Leibniz's rule $X(f g)=X(f) g+f X(g)$. Set $f_{i}:=X\left(x_{i}\right)$. Then it turns out that $X$ equals the derivation $Y:=\sum_{i=1}^{n} f_{i} \frac{\partial}{\partial x_{i}}$ defined in the natural way. Indeed, by Leibniz's rule and linearity the derivation $Z:=X-Y$ vanishes on polynomials. Moreover, by Leibniz's rule, $Z$ maps $M^{d}$ into $M^{d-1}$, hence $Z$ is continuous with respect to the metric above. Since the polynomials are dense in $K[[\mathbf{x}]]$, we find that $Z$ is identically zero, hence $X$ equals $Y$ as claimed.

The order ord $X$ of a derivation $X$ is defined as $-1+\min _{i}$ ord $X\left(x_{i}\right)$. Thus we have ord $X(f) \geq \operatorname{ord} X+\operatorname{ord} f$, and the elegance of this relation explains the term -1 in the definition of ord $X$. Note that the minimal possible order of a derivation is -1 , for derivations of the form $\sum_{i} c_{i} \frac{\partial}{\partial x_{i}}+$ higher order terms with not all $c_{i} \in K$ equal to 0 . The $K$-space Der $K[[\mathbf{x}]]$ of all formal vector fields forms a Lie algebra with respect to the bracket $[X, Y]:=X \circ Y-Y \circ X$, and we have ord $[X, Y] \geq \operatorname{ord} X+\operatorname{ord} Y$. In particular, the derivations of order at least 0 form a subalgebra of Der $K[[\mathbf{x}]]$.

We now come to the central definition of this paper.

Definition 1.1 A Lie algebra of vector fields in $n$ variables is a subalgebra $L$ of Der $K[[\mathbf{x}]]$, and it is called transitive if the subspace of $L$ consisting of elements of non-negative order in $L$ has codimension $n$ in $L$.

Remark 1.2 This is equivalent to the condition that for every vector $\left(c_{1}, \ldots, c_{n}\right) \in K^{n}$ there is a vector field in $L$ of the form $\sum c_{i} \frac{\partial}{\partial x_{i}}+$ higher order terms.

By the above, this subspace $L_{0}$ is then a subalgebra. Note that $n$ is the maximal possible codimension of $L_{0}$ in $L$ : any $n+1$-tuple of derivations has a suitable linear combination $X$ where all of the coefficients of the lowest-order terms $\frac{\partial}{\partial x_{1}}, \ldots, \frac{\partial}{\partial x_{n}}$ cancel out, so that $X$ has order at least 0 .

To justify the terminology transitive assume that $K=\mathbb{R}$ and that all elements $X$ of $L$ are convergent in the sense that all $X\left(x_{i}\right)$ are convergent power series near the origin. Then each $X$ can be integrated, and transitivity means that the union, over all $X \in L$, of the integral curves through the origin in $\mathbb{R}^{n}$ contains an open neighbourhood of the origin. Hence the corresponding infinitesimal group acts locally transitively near the origin, and $L_{0}$ is the Lie algebra of the isotropy group of the origin.

Lie's work and the subsequent work by various other authors to be described below has as a principal aim the classification of Lie algebras of vector fields up to coordinate changes. In our present set-up, this means the following. If $\phi$ is an automorphism of the $K$-algebra $K[[\mathbf{x}]]$ and $X$ is a derivation, then $X^{\phi}:=\phi X \phi^{-1}$ is again a derivation. Moreover, we have $[X, Y]^{\phi}=\left[X^{\phi}, Y^{\phi}\right]$, so that if $L$ is a Lie algebra of vector 
fields, then so is $L^{\phi}$. Thus the classification problem becomes: classify subalgebras of Der $K[[\mathbf{x}]]$ up to automorphisms.

Example 1.3 Let us do the classification of finite-dimensional Lie algebras of vector fields in one variable $x$. Let $L$ be such a Lie algebra, and let $S \subseteq\{-1,0,1,2, \ldots\}$ be the set of orders of elements of $L$. Clearly $|S|=\operatorname{dim} L$, so $S$ is finite.

Now note that the commutator of $X=\left(x^{d}+\right.$ h.o.t.) $\frac{\partial}{\partial x}$ (of order $\left.d-1\right)$ and $Y=\left(x^{d}+\right.$ h.o.t) $\frac{\partial}{\partial y}$ (of order $\left.e-1\right)$ equals $\left((e-d) x^{d+e-1}+\right.$ h.o.t.), and that this is of order $(d-1)+(e-1)$ if $d$ and $e$ are not equal. Hence if $2 \leq d<e$, then the vector fields $Y,[X, Y],[X,[X, Y]], \ldots$ have orders

$$
e-1<(d-1)+(e-1)<2(d-1)+(e-1)<\cdots .
$$

We conclude that $S$ contains at most one positive number.

Let $X \in L$ be of minimal order $d$ in $L$. There are three cases to be considered:

$d>0$ By the argument above, $S=\{d\}$ and $L=\langle X\rangle_{K}$, and the classification boils down to the classification of a single vector field. In one variable this is straightforward: after a coordinate change, $L$ equals $\left\langle x^{d} \frac{\partial}{\partial x}\right\rangle$ (and for distinct $d$ s these Lie algebras are not equivalent).

$d=0$ After a coordinate change, we may assume that $X=x \frac{\partial}{\partial x}$. Let $\operatorname{ad}(X)$ denote the linear map $L \rightarrow L, Y \mapsto[X, Y]$. If $Y=\left(\sum_{i=2}^{\infty} c_{i} x^{i}\right) \frac{\partial}{\partial x}$ is a second element of $L$, then $\operatorname{ad}(X)^{k} Y=\sum_{i=2}^{\infty}(i-1)^{k} c_{i} \frac{\partial}{\partial x}$. Using Vandermonde determinants one sees that if $c_{i}$ is non-zero for infinitely many $i$, then these vector fields for $k=0,1,2,3, \ldots$ are linearly independent, which contradicts the assumption that $L$ is finite-dimensional. Hence only finitely many of the $c_{i}$ are non-zero, and then the same Vandermonde argument shows that $x^{i} \frac{\partial}{\partial x} \in L$ for all $i$ with $c_{i}$ non-zero. By the argument in the previous case, there can only be one such $c_{i}$. Hence $L=\left\langle x \frac{\partial}{\partial x}, x^{i} \frac{\partial}{\partial x}\right\rangle_{K}$ for a single $i>1$, and for distinct $i$ these Lie algebras are not equivalent under coordinate changes (although they are isomorphic as abstract Lie algebras).

$d=-1$ In this case, for any $Y \in L$ of non-negative order, we have $\operatorname{ord}[X, Y]=$ $\operatorname{ord}(Y)-1$. Hence $S$ is an interval $\{-1,0, \ldots, e\}$ for some $e \leq 1$. If $e=-1$, then $L$ is spanned by $X$, and after a coordinate change we have $L=\left\langle\frac{\partial}{\partial x}\right\rangle_{K}$. If $e=0$, then $L$ contains an element $Y$ of order 0 . After a coordinate change we may assume that $Y=x \frac{\partial}{\partial x}$, and then an argument as in the previous case shows that $L=\left\langle\frac{\partial}{\partial x}, x \frac{\partial}{\partial x}\right\rangle_{K}$. Finally, if $e=1$, then we may again assume that $L$ contains $x \frac{\partial}{\partial x}$, and by a similar argument as before we have $L=\left\langle\frac{\partial}{\partial x}, x \frac{\partial}{\partial x}, x^{2} \frac{\partial}{\partial x}\right\rangle_{K}$.

Among the Lie algebras just found, only those with $d=-1$ are transitive. The largest one among them is isomorphic to $\mathfrak{s l}_{2}(K)$, the algebra of trace-zero $2 \times 2$-matrices with the commutator as Lie bracket. In fact, it can also be derived as follows (see, for instance, [23, Example 1.58]): let the group $\mathrm{SL}_{2}$ act on the projective line $\mathbb{P}^{1} K$ via Möbius transformations. This gives rise to a map from $\mathfrak{s l}_{2}$ into vector fields on the usual affine chart $\mathbb{A}^{1} K \subseteq \mathbb{P}^{1} K$, and with a suitable choice of coordinate these vector fields are $E=-\frac{\partial}{\partial x}, H=-2 x \frac{\partial}{\partial x}, F=x^{2} \frac{\partial}{\partial x}$, satisfying the familiar commutation rules 


$$
[H, E]=2 E, \quad[H, F]=-2 F, \quad[E, F]=H .
$$

Elements with these commutation rules are called a Chevalley basis of $\mathfrak{s l}_{2}$. The subalgebra spanned by $E$ and $H$ corresponds to the action of the group of upper triangular matrices (scaling and translation but no inversion, in Möbius terminology), and the subalgebra spanned by the $E$ corresponds to the group of translations only.

In a larger number of variables, the problem of classifying all Lie algebras $L$ of vector fields up to coordinate changes turns out to be intractable, already for the simple reason that classifying a single vector field vanishing at the origin up to coordinate changes (without, for instance, any restrictions on its linear part as in Poincaré-Dulac theory) is intractable. Hence we will from now on restrict our attention to transitive algebras $L$, whose structure turns out to be very beautiful. The remainder of this paper is organised as follows. In Sect. 2 we describe Lie's classification of transitive Lie algebras of vector fields in two variables, and their appearance as symmetry algebras of scalar ordinary differential equations. In Sect. 3 we present the beautiful work by Guillemin-Sternberg and by Blattner that relates transitive Lie algebras to pairs of a Lie algebra and a subalgebra. In Sect. 4 we describe the classification of the primitive ones among these pairs, largely due to Morozov and Dynkin. Finally, in Sect. 5 we go back to an observation by Lie on Lie algebras of vector fields with "nice" coefficients, which inspires two challenging research problems that conclude the paper.

\section{Lie: Transitive Lie Algebras in Dimension Two}

Towards the end of the nineteenth century, Lie classified finite-dimensional transitive Lie algebras of vector field in two variables [20]. To achieve this, he divided these Lie algebras into two classes, namely, the primitive ones and the imprimitive ones. In modern Lie-algebraic terminology these notions are defined as follows.

Definition 2.1 Let $L$ be a transitive subalgebra of Der $K\left[\left[x_{1}, \ldots, x_{n}\right]\right]$, and let $L_{0}$ be its subalgebra of elements with non-negative order. Then $L$ is called imprimitive if there exists a subalgebra $L^{\prime}$ of $L$ that lies strictly between $L_{0}$ and $L$.

As with transitivity, this terminology is best explained in the setting where $K=\mathbb{R}$ and the elements of $L$ are convergent. If $L^{\prime}$ is a subalgebra strictly between $L_{0}$ and $L$, of codimension $m, 0<m<n$ in $L$ say, then integrating $L^{\prime}$ (using a weak version of Frobenius's theorem on vector fields in involution; see for instance [23, Theorem 1.40]) yields a foliation of a neighbourhood of 0 in $\mathbb{R}^{n}$ with sheets of dimension $n-m$, and these sheets are permuted by all (local) one-parameter subgroups corresponding to elements in $L$. Thus these sheets form a system of imprimitivity. In the case of two variables, a subalgebra $L^{\prime}$ as above corresponds to a foliation of a neighbourhood of the origin in $\mathbb{R}^{2}$ by curves permuted by the infinitesimal group corresponding to $L$ (eine invariante Kurvenschare in Lie's terminology), and $L$ is primitive if no such foliation exists.

We now give Lie's classification of primitive and imprimitive Lie algebras in two variables $x, y$, where we adopt Lie's notation $p:=\frac{\partial}{\partial x}, q:=\frac{\partial}{\partial y}$. 
Table 1 The primitive Lie algebras in two variables

\begin{tabular}{lll}
\hline Type & Lie algebra & Label \\
\hline$(5)$ & $\langle p, q, x q, x p-y q, y p\rangle$ & A3 \\
$(6)$ & $\langle p, q, x q, x p-y q, y p, x p+y q\rangle$ & A2 \\
$(8)$ & $\left\langle p, q, x q, x p-y q, y p, x p+y q, x^{2} p+x y q, x y p+y^{2} q\right\rangle$ & A1 \\
\hline
\end{tabular}

The third column of Table 1 contains the the label given to these algebras in [11] and [20]. The type will be explained in the next section

Theorem 2.2 (Lie [20]) Every finite-dimensional, primitive Lie algebra in two variables over an algebraically closed field $K$ of characteristic zero equals one of the Lie algebras in Table 1, up to a formal coordinate change.

In fact, if $K=\mathbb{C}$, then "formal" in this theorem can be replaced by "convergent"; see Remark 3.6 below.

Theorem 2.3 (Lie [20]) Every imprimitive (but transitive) Lie algebra in two variables over an algebraically closed field $K$ of characteristic zero can be moved by a formal coordinate change into one of the 16 (families of) Lie algebras in Table 2.

The classification over the real numbers is slightly more involved; see [12].

Remark 2.4 There is some redundancy in Table 2. For instance, the Lie algebra $L=$ $\langle p, x p+q\rangle$ of type $(2,1)$, case 1 , can also be brought into a Lie algebra in the family of type $(1,1)$. To see this, consider the coordinate change $x=v \exp (-u)$ and $y=-u$ with inverse $u=-y$ and $v=x \exp (-y)$. Under this coordinate change, we have

$$
\begin{gathered}
p=\frac{\partial u}{\partial x} \frac{\partial}{\partial u}+\frac{\partial v}{\partial x} \frac{\partial}{\partial v}=\exp (u) \frac{\partial}{\partial v} \\
q=\frac{\partial u}{\partial y} \frac{\partial}{\partial u}+\frac{\partial v}{\partial y} \frac{\partial}{\partial v}=-\frac{\partial}{\partial u}-v \frac{\partial}{\partial v} \\
x p+q=-\frac{\partial}{\partial u}
\end{gathered}
$$

so that the coordinate change moves the Lie algebra $L$ into the member of the family in the first row with $\alpha$ running through the set $\{1\}$ and $r_{1}=0$. A similar computation moves the Lie algebra of type $(3,1)$, case 2 , into a Lie algebra of type $(1,3)$. This explains the lack of labels in the third row for these two Lie algebras. This redundancy will be explained in the next section.

In the following section we will recast these classifications in modern terminology, explaining the first column in both tables in the process. But first let us quickly review why Lie was interested in classifying Lie algebras of vector fields in two variables. This discussion will not be needed in the following sections.

Let $A=K[[x, y]]\left[y^{\prime}, y^{\prime \prime}, \ldots\right]$ be the ring of polynomials in the (algebraically independent) variables $y^{\prime}=y^{(1)}, y^{\prime \prime}=y^{(2)}, \ldots$ with coefficients that are formal power series in the variables $x, y=y^{(0)}$. An element of $A$ represents the left-hand-side of a 
Table 2 The non-primitive transitive Lie algebras in two variables

\begin{tabular}{|c|c|c|c|}
\hline Type & Case & Realisation & Label \\
\hline$(1,1)$ & & $\begin{array}{l}\left\langle p, x^{i} \exp (\alpha x) q\right\rangle \\
\quad \text { where } i=0, \ldots, r_{\alpha} \geq 0 \\
\text { and } \alpha \text { in a non-empty finite set }\end{array}$ & $\mathrm{B} \beta 1, \mathrm{D} 1, \mathrm{D} 2$ \\
\hline$(1,2)$ & & $\begin{array}{l}\left\langle p, y q, x^{i} \exp (\alpha x) q\right\rangle \\
\quad \text { where } i=0, \ldots, r_{\alpha} \geq 0 \\
\text { and } \alpha \text { in a non-empty finite set }\end{array}$ & $\mathrm{B} \beta 2, \mathrm{C} 2$ \\
\hline$(1,3)$ & & $\left\langle p, q, 2 y q,-y^{2} q\right\rangle$ & $\mathrm{C} 5$ \\
\hline$(2,1)$ & 1 & $\begin{array}{l}\langle p, x p+q\rangle \\
\text { For a different } \mathfrak{h}_{1}, \text { this is }(1,1) .\end{array}$ & \\
\hline$(2,1)$ & 2 & $\begin{array}{l}\left\langle p, x p, x^{i} q\right\rangle, \text { where } i=0, \ldots, r \geq 0 \\
\text { For } r=0 \text { and a different } \mathfrak{h}_{1}, \text { this is }(1,2)\end{array}$ & $\mathrm{B} \gamma 1$ \\
\hline$(2,2)$ & 1 & $\left\langle p, x p-\lambda y q, x^{i} q\right\rangle$, where $i=0, \ldots, r \geq 0$ and $\lambda \neq 0$ & $\mathrm{~B} \gamma 2, \mathrm{C} 8, \mathrm{D} 3$ \\
\hline$(2,2)$ & 2 & $\begin{array}{l}\left\langle p, x p+\left((r+1) y+x^{r+1}\right) q, x^{i} q\right\rangle \\
\quad \text { where } i=0, \ldots, r \geq 0\end{array}$ & $\mathrm{~B} \gamma 3$ \\
\hline$(2,2)$ & 3 & $\left\langle p, x p, y q, x^{i} q\right\rangle$, where $i=0, \ldots, r \geq 0$ & $\mathrm{~B} \gamma 4, \mathrm{C} 3$ \\
\hline$(2,3)$ & & $\left\langle p, x p, q, 2 y q,-y^{2} q\right\rangle$ & C6 \\
\hline$(3,1)$ & 1 & $\left\langle p, 2 x p+q,-x^{2} p-x q\right\rangle$ & $\mathrm{B} \delta 1$ \\
\hline$(3,1)$ & 2 & $\begin{array}{l}\left\langle p, 2 x p,-x^{2} p, q\right\rangle \\
\text { For a different } \mathfrak{h}_{1} \text {, this is }(1,3) .\end{array}$ & \\
\hline$(3,1)$ & 3 & $\left\langle p, 2 x p-q,-x^{2} p+x q, q\right\rangle$ & $\mathrm{B} \delta 2$ \\
\hline$(3,2)$ & 1 & $\left\langle p, 2 x p-2 y q,-x^{2} p+(1+2 x y) q\right\rangle$ & $\mathrm{C} 9$ \\
\hline$(3,2)$ & 2 & $\begin{array}{l}\left\langle p, 2 x p+r y q,-x^{2} p-r x y q, x^{i} q\right\rangle \\
\quad \text { where } i=0, \ldots, r \geq 1\end{array}$ & $\mathrm{~B} \delta 3$ \\
\hline$(3,2)$ & 3 & $\begin{array}{l}\left\langle p, 2 x p+r y q,-x^{2} p-r x y q, y q, x^{i} q\right\rangle, \\
\quad \text { where } i=0, \ldots, r \geq 0 \\
\text { For } r=0 \text { and a different } \mathfrak{h}_{1} \text { this is }(2,3) \text {. }\end{array}$ & $\mathrm{B} \delta 4$ \\
\hline$(3,3)$ & & $\left\langle p, 2 x p,-x^{2} p, q, 2 y q,-y^{2} q\right\rangle$ & $\mathrm{C} 7$ \\
\hline
\end{tabular}

This table differs from Lie's table in that the origin $(0,0)$ is always a regular point. Moreover, whenever an $\mathfrak{s l}_{2}$ occurs, its Chevalley basis is contained in the basis given in our table. The third column can be used for translation between this table and Lie's table in [20]. The type will be explained in the next section

scalar ordinary differential equation, with $x$ playing the role of independent variable and $y, y^{\prime}, \ldots$ playing the role of the dependent variable and its derivatives with respect to $x$. On $A$ one defines the total derivative with respect to $x$ as the derivation

$$
D_{x}=\frac{\partial}{\partial x}+\sum_{i=0}^{\infty} y^{(i+1)} \frac{\partial}{\partial y^{(i)}} .
$$

A vector field $X$ in two variables $x, y$ has a unique extension, still denoted by $X$, to a $K$-linear derivation on $A$ for which $\left[X, D_{x}\right]$ equals $Q D_{x}$ for some $Q \in A$. This exten- 
sion is called the prolongation of $X$ (for this short characterisation of the prolongation see [24, Lemma 2.4]). Since the effect of $X$ on power series in $x$ and $y$ is already prescribed, and since the prolongation is to satisfy Leibniz's rule, the prolongation is determined by its value on $y^{\prime}, y^{\prime \prime}, \ldots$ Writing $f:=X(x)$ and $g:=X(y)$ and using short-hand notation such as $f_{x y}$ for $\frac{\partial^{2} f}{\partial x \partial y}$, we find

$$
\begin{aligned}
Q= & \left(Q D_{x}\right) x=\left[X, D_{x}\right](x)=X(1)-D_{x}(X(x))=-f_{x}-f_{y} y^{\prime} \\
X\left(y^{\prime}\right)= & X\left(D_{x}(y)\right)=D_{x}(X(y))+\left[X, D_{x}\right](y) \\
= & g_{x}+g_{y} y^{\prime}+Q y^{\prime}=g_{x}+\left(g_{y}-f_{x}\right) y^{\prime}-f_{y}\left(y^{\prime}\right)^{2} \\
X\left(y^{\prime \prime}\right)= & X\left(D_{x}\left(y^{\prime}\right)\right)=D_{x}\left(X\left(y^{\prime}\right)\right)+\left[X, D_{x}\right]\left(y^{\prime}\right) \\
= & g_{x x}+\left(2 g_{x y}-f_{x x}\right) y^{\prime}+\left(g_{y y}-f_{x y}\right)\left(y^{\prime}\right)^{2}+\left(g_{y}-f_{x}\right) y^{\prime \prime} \\
& -f_{x y}\left(y^{\prime}\right)^{2}-f_{y y}\left(y^{\prime}\right)^{3}-2 f_{y} y^{\prime} y^{\prime \prime}+Q y^{\prime \prime} \\
= & g_{x x}+\left(2 g_{x y}-f_{x x}\right) y^{\prime}+\left(g_{y y}-2 f_{x y}\right)\left(y^{\prime}\right)^{2}+\left(g_{y}-2 f_{x}\right) y^{\prime \prime} \\
& -f_{y y}\left(y^{\prime}\right)^{3}-3 f_{y} y^{\prime} y^{\prime \prime}
\end{aligned}
$$

etc. The idea behind this definition is that the prolongation of $X$ describes the vector field induced by $X$ on higher jet spaces, where $y$ is considered the dependent variable and $x$ the independent variable: if $K=\mathbb{R}$ and $t \mapsto(x(t), y(t))$ is an integral curve of $X$ with $\frac{\mathrm{d} x}{\mathrm{~d} t}\left(t_{0}\right) \neq 0$, then near $t_{0}$ we may write $t$ and $y$ as functions of $x$, repeatedly differentiate $y$ with respect to $x$, and rewrite those derivatives as functions of $t$. Then $t \mapsto\left(x(t), y(t), y_{x}(t), y_{x x}(t), \ldots\right)$ is the unique integral curve of the prolongation of $X$ that projects to that of $X$.

Now we come to the central connection between Lie algebras of vector fields and ordinary differential equations.

Definition 2.5 Let $P$ be an element of $A$ of the form $y^{(m)}-Q$ with $m \geq 2$ a natural number and $Q$ an element of $A$ involving none of the variables $y^{(i)}$ with $i \geq m$, so that $P=0$ is an explicit o.d.e. of order $m \geq 2$. Define $L(P)$ as the $K$-space of all vector fields $X \in \operatorname{Der} K[[x, y]]$ whose prolongation satisfies $P \mid X(P)$, that is, the polynomial $X(P)$ in $y^{\prime}, y^{\prime \prime}, \ldots$ is divisible by $P$ in the ring $A$. The space $L(P)$ is called the algebra of Lie (point) symmetries of the ordinary differential equation $P=0$.

For non-explicit $P$, the definition of $L(P)$ is slightly more complicated; see [24, Definition 2.5] in an algebraic setting and the standard reference [23, Chapter 2] in the smooth setting (including the case of multiple independent variables); in what follows we assume that $P$ is as in the definition. An easy computation shows that $L(P)$ is, indeed, a Lie algebra. The rationale for this definition can again be found in jet spaces: A local solution $y=y(x)$ of $P=0$ gives rise to a curve in higher jet spaces, and applying the infinitesimal transformation corresponding to (the prolongation of) $X$ to such a "jet curve" should yield a curve on which $P$ vanishes, as well, i.e., another solution curve.

For $P$ explicit of order at least two, one can show that $L(P)$ is a finite-dimensional Lie algebra over over $K$ (with a uniform bound on the dimension; see [23, Exercise 2.27] and references there). Having a classification of these Lie algebras at hand, one 
may try to solve $P=0$ by making a change of coordinates that brings $L(P)$ into normal form, and analysing the solutions of equations $P$ for which $L(P)$ is in normal form. This beautiful proposal by Lie involves steps that are difficult from an algebraic and algorithmic perspective. In fact, whether $L(P)$ is transitive can be determined algorithmically and if it is transitive, the relevant entry in Table 1 can be determined algorithmically, as well $[6,25,26]$, but the coordinate change bringing the Lie algebra in normal form can be arbitrarily complicated, and it is not clear when coordinate changes involving only "elementary functions" exist. Nevertheless, special cases of this idea are presently used in computer software for solving o.d.e.s.

Remark 2.6 Note that $L(P)$ may very well not be transitive. In fact, apart from proposing the solution strategy above, Lie also strongly advocated the use of a single known vector field in $L(P)$ for trying to solve $P$. Numerous examples of o.d.e.s of orders one, two, and three where this works can be found in [19].

We conclude this section with two examples, one well-known "direct" example and one "inverse" example.

Example 2.7 First consider the o.d.e. $P=0$ with $P=y^{\prime \prime}$. Let $X=f \frac{\partial}{\partial x}+g \frac{\partial}{\partial y}$ be a vector field, and denote its prolongation by $X$, as well. For $X$ to be in $L(P)$, the expression (1) for $X(P)$ must be a multiple of $P$. This is equivalent to the system

$$
\begin{array}{ll}
g_{x x}=0 & 2 g_{x y}-f_{x x}=0 \\
f_{y y}=0 & g_{y y}-2 f_{x y}=0
\end{array}
$$

of linear p.d.e.s for $f$ and $g$. This is the so-called determining system for $L(P)$. For general $P$, this determining system is a typically over-determined system of linear p.d.e.s for the coefficients $f, g$ whose solution space is the algebra of Lie symmetries. Such systems can be analysed with Buchberger-type algorithms [25,29]. In fact, the relevant Janet Bases for systems of linear p.d.e.s predate Buchberger's Gröbner bases. For a good recent overview of their theory and implementations see [28].

In our particular case, the solution space to the determining system is spanned by $(f, g)=\left(x^{2}, x y\right)$ and $(f, g)=\left(x y, y^{2}\right)$ together with all pairs $(f, g)$ of (affine- $)$ linear polynomials. We conclude that $L(P)$ is the Lie algebra of type (8) in Table 1 . This fact has the following geometric interpretation: the solutions of the o.d.e. $y^{\prime \prime}=0$ are all (non-vertical) straight lines, and the Lie algebra of type (8) is the Lie algebra coming from the action of $\mathrm{SL}_{3}$ on the projective plane by means of projective linear transformations. These transformations permute the collection of all straight lines. Note that here the infinitesimal (or at least local) character of the algebra of Lie symmetries becomes apparent: clearly, a projective linear transformation may well transform a non-vertical line in the plane into a vertical one, or even to the line at infinity. But given one solution (strictly speaking again local), there is an open neighbourhood of the identity in $\mathrm{SL}_{3}$ that maps map the given solution to other solutions.

Example 2.8 Let us try to find an explicit o.d.e. $P=0$ of order $m \geq 2$ such that $L(P)$ is the Lie algebra of type (5) in Table 1. For this, we compute the prolongations of 
some of the vector fields in $L(P)$ : First, the prolongation of $\frac{\partial}{\partial x}$ is just $\frac{\partial}{\partial x}$, and $\frac{\partial P}{\partial x}$ can only be a multiple of $P$ if it is zero. Hence $P=0$ is an autonomous o.d.e. Second, the prolongation of $\frac{\partial}{\partial y}$ is just $\frac{\partial}{\partial y}$, and we find that $P$ does not depend (explicitly) on $y$. Third, the prolongation of $x \frac{\partial}{\partial y}$ equals $x \frac{\partial}{\partial y}+\frac{\partial}{\partial y^{\prime}}$. Since $P$ does not depend on $y$ and since the order of $P$ is at least two we find that $P$ does not depend on $y^{\prime}$ either. Hence the only second-order explicit o.d.e. whose algebra of Lie symmetries contains the vector fields so far is $y^{\prime \prime}=0$, and we already know its algebra of Lie symmetries to be the entire algebra of type (8) in Table 1. In particular, if the algorithm of [6] says that the algebra of Lie symmetries of a given second-order o.d.e. is of type (8), then it can be transformed into $y^{\prime \prime}=0$ by a coordinate change (note that we are being slightly sloppy here, since formal coordinate changes in $x$ and $y$ do not necessarily map polynomial o.d.e.s to polynomial o.d.e.s).

Fourth, an easy induction shows that for $X=y \frac{\partial}{\partial x}$ the expression $X\left(y^{(m)}\right), m \geq 2$ is a linear combination of the monomials

$$
y^{(1)} y^{(m)}, y^{(2)} y^{(m-1)}, \ldots, y^{\left(\left\lfloor\frac{m+1}{2}\right\rfloor\right)} y^{\left(\left\lceil\frac{m+1}{2}\lceil)\right.\right.},
$$

each with a non-zero coefficient. Applying $X$ to $P=y^{(m)}-Q\left(y^{(2)}, \ldots, y^{(m-1)}\right)$ and replacing $y^{(m)}$ by $Q$ in the result leaves, for instance, a non-zero multiple of $y^{(2)} y^{(m-1)}$ that cannot be cancelled by any of the other terms. Thus there exists no explicit polynomial o.d.e. whose algebra of symmetries equals the algebra (5) in Table 1. On the other hand, if one enlarges the algebra $A$ to include more complicated o.d.e.s, then there do exist examples with $L(P)$ equal to the Lie algebra (5), such as

$$
P:=y^{(4)}-\frac{5\left(y^{(3)}\right)^{2}}{3 y^{(2)}}-\left(y^{(2)}\right)^{5 / 3}=0 .
$$

To check this, one needs only verify that the prolongation of $y \frac{\partial}{\partial x}$ is in $L(P)$ and that the prolongation of $x \frac{\partial}{\partial x}$ is not-otherwise $L(P)$ would include Lie algebra (6) of Table 1 .

\section{Guillemin-Sternberg and Blattner: The Realisation Theorem}

We return to transitive Lie algebras of vector fields in $n$ variables. In their groundbreaking work [14] Guillemin and Sternberg extracted the following fundamental properties of such a Lie algebra $L \subseteq$ Der $K[[\mathbf{x}]]$ and its isotropy algebra $L_{0} \subseteq L$ consisting of all vector fields in $L$ of non-negative order: First, $L_{0}$ has codimension $n$ in $L$ - this was the defining condition for $L$ to be transitive. Second, the only ideal of $L$ contained in $L_{0}$ is $\{0\}$; here an ideal of $L$ is a subspace $I$ with the property that the Lie bracket $[X, I]$ lies in $I$ for all $X$ in $L$. This second property can be seen as follows. Suppose that an $L$-ideal $I$ contains a non-zero element $Y_{0}=\sum_{i=1}^{n} f_{i} \frac{\partial}{\partial x_{i}}$ of non-negative order $d$. Let $f_{i}$ be of minimal order $d+1 \geq 1$, and let $x_{j}$ be a variable such that the degree- $(d+1)$ part of $f$ really depends on $x_{j}$. Since $L$ is transitive, it contains an element $X$ of the form $\frac{\partial}{\partial x_{j}}+Z$ with ord $Z \geq 0$. Then 


$$
Y_{1}:=\left[X, Y_{0}\right]=\left[\frac{\partial}{\partial x_{j}}+Z, Y_{0}\right]=\left[\frac{\partial}{\partial x_{j}}, Y_{0}\right]+\left[Z, Y_{0}\right]
$$

Here the order of $\left[Z, Y_{0}\right]$ is at least $d$, while $\left[\frac{\partial}{\partial x_{j}}, Y_{0}\right]$ is by construction non-zero with order $d-1$. Hence $Y_{1}$ has order $d-1$, and it belongs again to the ideal $I$. Continuing in this manner, one finds that $I$ contains elements of order -1 and is therefore not contained in $L_{0}$.

The striking insight by Guillemin and Sternberg is that the two conditions on $\left(L, L_{0}\right)$ suffice to determine a transitive Lie algebra in $n$ variables. More precisely, in what follows we will work with so-called transitive pairs.

Definition 3.1 A transitive pair is a pair $(\mathfrak{g}, \mathfrak{h})$ of a Lie algebra $\mathfrak{g}$ over $K$ with a subalgebra $\mathfrak{h}$ of finite codimension in $\mathfrak{g}$; this is then called the codimension of the transitive pair. The pair is called effective if $\mathfrak{h}$ contains no non-zero $\mathfrak{g}$-ideal. The pair is called primitive if $\mathfrak{h}$ is a maximal (strict) subalgebra of $\mathfrak{g}$. A realisation of a transitive pair $(\mathfrak{g}, \mathfrak{h})$ is a Lie algebra homomorphism $\phi: \mathfrak{g} \rightarrow \operatorname{Der} K\left[\left[x_{1}, \ldots, x_{n}\right]\right]$ such that $n=\operatorname{codim}_{\mathfrak{g}} \mathfrak{h}$ and such that $\mathfrak{h}$ consists exactly of those elements of $\mathfrak{g}$ that are mapped by $\phi$ to vector fields of non-negative order.

Remark 3.2 Transitive pairs are the infinitesimal counterparts of homogeneous spaces $G / H$ where $H$ is a closed subgroup of a Lie group $G$. Such spaces, and in particular the symmetric spaces among them, are ubiquitous in differential geometry; see [15]. More general manifolds-with-extra-structure, locally modelled on homogeneous spaces and in some sense generalising Riemannian manifolds, are the object of study in [30]. Again, transitive pairs $(\mathfrak{g}, \mathfrak{h})$ serve as indispensable infinitesimal models.

Loosely speaking, Guillemin and Sternberg proved that realisations of transitive pairs exist and are unique up to coordinate changes; here is the precise statement.

Theorem 3.3 (Realisation Theorem, Guillemin-Sternberg [14]) Every transitive pair has a realisation. Moreover, if $\phi_{1}, \phi_{2}$ are realisations of the same transitive pair $(\mathfrak{g}, \mathfrak{h})$, then there exists a unique coordinate change $\psi: K[[\mathbf{x}]] \rightarrow K[[\mathbf{x}]]$ such that, for all $X \in \mathfrak{g}$, we have $\phi_{2}(X)=\psi \phi_{2}(X) \psi^{-1}$. Furthermore, the kernel $\phi^{-1}(0)$ of any realisation $\phi$ of the transitive pair $(\mathfrak{g}, \mathfrak{h})$ is equal to the largest $\mathfrak{g}$-ideal contained in $\mathfrak{h}$. In particular, the realisation is an embedding if and only if the pair is effective.

In view of this realisation theorem, Lie's classification in the previous section can be understood as a classification of all finite-dimensional and effective transitive pairs $(\mathfrak{g}, \mathfrak{h})$ of codimension 2 , up to natural isomorphisms between such pairs. This modern view on Lie's classification helps in understanding (and even re-doing) it. Assume that the pair is not primitive (for primitive Lie algebras, see the following section). Then there exists a subalgebra $\mathfrak{l}$ such that $\mathfrak{g} \supset \mathfrak{l} \supset \mathfrak{h}$, where the inclusions are proper. In general, the transitive pairs $(\mathfrak{g}, \mathfrak{l})$ and $(\mathfrak{l}, \mathfrak{h})$ will no longer be effective. Denote the largest $\mathfrak{g}$-ideal contained in $\mathfrak{l}$ by $\mathfrak{i}$ and the largest $\mathfrak{l}$-ideal contained in $\mathfrak{h}$ by $\mathfrak{j}$. The quotients $(\mathfrak{g} / \mathfrak{i}, \mathfrak{l} / \mathfrak{i})$ and $(\mathfrak{l} / \mathfrak{j}, \mathfrak{h} / \mathfrak{j})$ are both effective pairs of codimension 1 , and hence each is isomorphic to one of the three transitive pairs found in Example 1.3. Hence in total, there are nine possibilities for the pair $(\operatorname{dim} \mathfrak{g} / \mathfrak{i}, \operatorname{dim} l / \mathfrak{j})$. This pair is called the 
type of the triple $(\mathfrak{g}, \mathfrak{l}, \mathfrak{h})$. In our Table 2 , the type is recorded in the first column but $\mathfrak{l}$ is suppressed. Sometimes, various intermediate subalgebras $\mathfrak{l}$ can be chosen, and the resulting triples may be of different types. As a consequence, an effective pair $(\mathfrak{g}, \mathfrak{h})$ may occur several times in the classification. When this happens, there is a reference to an earlier entry of the list, to which it is isomorphic. This explains the redundancy observed in Remark 2.4.

\subsection{Blattner's Proof of the Realisation Theorem}

Guillemin and Sternberg's original proof involves finding both the required formal vector fields and the coordinate change relating two realisations recursively, "coefficient by coefficient". A few years later, Blattner came up with a beautiful coordinate-free proof [1], where the uniqueness follows from the universal property of certain representations. I will now spend a few paragraphs explaining the proof, using basic Lie algebra theory as can be found, for instance, in [16]. Making his proof explicit leads to a Realisation Formula below. This formula will not be used in the next section, but it will be used in the last section of this paper.

To sketch Blattner's proof, we fix a transitive pair $(\mathfrak{g}, \mathfrak{h})$ and we recall the concept of universal enveloping algebra $U(\mathfrak{g})$ of $\mathfrak{g}$. This is an associative algebra built from $\mathfrak{g}$ in the free-est possible way, subject to the condition that the Lie bracket between elements in $\mathfrak{g}$ coincides with their commutator in $U(\mathfrak{g})$. Formally, $U(\mathfrak{g})$ is the quotient of the tensor algebra $\bigoplus_{d=0}^{\infty} \mathfrak{g}^{\otimes d}$ generated by the vector space $\mathfrak{g}$ by the (two-sided) ideal generated by elements of the form

$$
X \otimes Y-Y \otimes X-[X, Y]
$$

with $X$ and $Y$ running through $\mathfrak{g}$. This expresses that, in $U(\mathfrak{g})$, the element $X \otimes Y-$ $Y \otimes X \in \mathfrak{g}^{\otimes 2}$ equals the Lie bracket $[X, Y] \in \mathfrak{g}^{\otimes 1}$ of the elements $X, Y$. The inclusion of $\mathfrak{g}=\mathfrak{g}^{\otimes 1}$ into the tensor algebra gives a map $\mathfrak{g} \rightarrow U(\mathfrak{g})$, and this linear map is, in fact, injective. For ease of exposition, we assume that $\mathfrak{g}$ is finite-dimensional, although this is not needed for Blattner's proof (and although the theory of transitive infinitedimensional Lie algebras is very rich; see [13] or, for the real $C^{\infty}$-case, [31]). Let $X_{1}, \ldots, X_{m}$ be a basis of $\mathfrak{g}$. Then an easy induction shows that, modulo the defining relations above, any monomial

$$
X_{i_{1}} \otimes \cdots \otimes X_{i_{d}}
$$

equals a linear combination of such monomials with the additional property that the index sequence is weakly increasing. Such monomials will be called ordered. Indeed, this linear combination will start with the signed ordered monomial $\pm X_{i_{\pi(1)}} \otimes \cdots \otimes X_{i_{\pi(d)}}$ (with $\pi$ a permutation rendering the indices weakly increasing) and continue with monomials of lower degree. Thus the ordered monomials span the universal enveloping algebra. The celebrated Poincaré-Birkhoff-Witt theorem (see, for instance, [16, Chapter V, Theorem 3]) states that they are, in fact, a basis of this algebra. By taking for $X_{1}, \ldots, X_{m-n}$ a basis of $\mathfrak{h}$ and for $Y_{1}:=X_{m-n+1}, \ldots, Y_{n}:=X_{m}$ a basis of a complementary subspace, one finds that every element in $U(\mathfrak{g})$ can be written in a unique way as 


$$
\sum_{\alpha \in \mathbb{N}^{n}} u_{\alpha} Y_{1}^{\alpha_{1}} \cdots Y_{n}^{\alpha_{n}}
$$

with only finitely many terms non-zero and with coefficients $u_{\alpha}$ in the universal enveloping algebra $U(\mathfrak{h}) \subseteq U(\mathfrak{g})$ of $\mathfrak{h}$. In the case where $\mathfrak{h}$ is zero-dimensional, this is just a restatement of the Poincaré-Birkhoff-Witt theorem. Note that we have left out the $\otimes-$ signs here, and just use juxtaposition to denote multiplication in $U(\mathfrak{g})$. This notational choice is especially important since other tensor products will soon play a role.

We will get back to this explicit description of $U(\mathfrak{g})$ as a left- $U(\mathfrak{h})$-module a bit later. For the moment, we will not need it—and this is the beauty of Blattner's proof. The universal enveloping algebra of $\mathfrak{g}$ plays the same role in the representation theory of $\mathfrak{g}$ as the the group algebra $\mathbb{C} G$ of a finite group $G$ plays in representation theory of $G$. In particular, a $\mathfrak{g}$-module (or a representation of $\mathfrak{g}$ as a Lie algebra over $K$ ) gives rise to a $U(\mathfrak{g})$-module (or a representation of $U(\mathfrak{g}$ ) as an associative algebra over $K$ ), and vice versa. Now Blattner introduces

$$
A:=\operatorname{Hom}_{U(\mathfrak{h})}(U(\mathfrak{g}), K)
$$

as a coordinate-free version of the algebra $K[[\mathbf{x}]]$ of formal power series; let me explain. First, $K$ is the trivial left- $\mathfrak{h}$-module, in which every element of $\mathfrak{h}$ acts as zero. As a consequence, all monomials in $U(\mathfrak{h})$ of positive degree act as zero, as well, while its unit element $1 \in \mathfrak{h}^{\otimes 0}=K$ acts as unit element (as one usually requires of associative-algebra representations). Second, $U(\mathfrak{g})$ is a $U(\mathfrak{h})$-module by left multiplication. Third, $A$ is the space of all $K$-linear maps $a: U(\mathfrak{g}) \rightarrow K$ such that $a(v u)=v a(u)$ for all $v \in U(\mathfrak{h})$ and $u \in U(\mathfrak{g})$. This is equivalent to the condition that $a(Y v)=0$ for all $Y \in \mathfrak{h}$.

One makes $A$ into a commutative algebra as follows. Let $\Delta: U(\mathfrak{g}) \rightarrow U(\mathfrak{g}) \otimes U(\mathfrak{g})$ denote the unique algebra homomorphism determined by $\Delta(X)=X \otimes 1+1 \otimes X$ for $X \in \mathfrak{g} \subseteq U(\mathfrak{g}) .{ }^{1}$ Then we define $a \cdot b$ as the the composition of the following sequence of maps:

$$
U(\mathfrak{g}) \stackrel{\Delta}{\rightarrow} U(\mathfrak{g}) \otimes U(\mathfrak{g}) \stackrel{a \otimes b}{\rightarrow} K \otimes K \rightarrow K
$$

where the last map is just the natural (multiplication) isomorphism $K \otimes K \rightarrow K$. A straightforward computation shows that $(a \cdot b)(Y v)=0$ for all $Y \in \mathfrak{h}$, so that $a \cdot b \in A$. The fact that $\cdot$ is commutative follows from the fact that for every $u \in U(\mathfrak{g})$, $\Delta(u) \in U(\mathfrak{g}) \otimes U(\mathfrak{g})$ is invariant under swapping the two tensor factors-the co-commutativity of $\Delta$. Similarly, the fact that $\Delta$ is co-associative, i.e., satisfies $(\Delta \otimes 1) \circ \Delta=$ $(1 \otimes \Delta) \circ \Delta$, implies that $\cdot$ is associative.

The algebra $A$ will serve as our coordinate-free model for the algebra of formal power series-we will describe an isomorphism below. Hence we want $\mathfrak{g}$ to act by derivations on $A$; this goes as follows. Given $X \in \mathfrak{g}$ and $a \in A$, we define a new

\footnotetext{
${ }^{1}$ This is the Lie algebra analogue of the natural homomorphism $\mathbb{C} G \rightarrow \mathbb{C} G \otimes \mathbb{C} G$ extending $g \mapsto g \otimes g$ for finite groups $G$, which allows one to see the tensor product of two representations of $G$ as a new representation of $G$, as opposed to a representation of $G \times G$.
} 
element $X a \in A$ by $(X a)(u)=a(u X)$. Note that $(X a)(v u)=a(v u X)=v a(u X)=$ $v(X a)(u)$ for $v \in U(\mathfrak{h})$, so that $X a$ does indeed lie in $A$. In this way, $A$ becomes a $\mathfrak{g}$-module, as a straightforward computation shows. Moreover, we have Leibniz's identity $X(a \cdot b)=X(a) \cdot b+a \cdot X(b)$ for all $a, b \in A$ and $X \in \mathfrak{g}$. Indeed, fixing $u \in \mathrm{U}(\mathfrak{g})$ and writing $\Delta u=\sum_{i} u_{i} \otimes w_{i}$, we find $\Delta(u X)=\sum_{i} u_{i} X \otimes w_{i}+\sum_{i} u_{i} \otimes w_{i} X$, and hence

$$
\begin{aligned}
(X(a \cdot b)) u & =(a \cdot b)(u X)=\sum_{i} a\left(u_{i} X\right) b\left(u_{i}\right)+\sum_{i} a\left(u_{i}\right) b\left(u_{i} X\right) \\
& =(X a \cdot b+a \cdot X b) u
\end{aligned}
$$

as claimed.

Thus we have $\mathfrak{g}$ acting by means of derivations on an algebra $A$. Now identifying $A$ with formal power series gives the existence of a realisation of the transitive pair $(\mathfrak{g}, \mathfrak{h})$. This identification goes as follows. Fix a basis $Y_{1}, \ldots, Y_{n}$ of a vector space complement of $\mathfrak{h}$ in $\mathfrak{g}$. We have already seen that every element of $U(\mathfrak{g})$ has a unique representation as $U(\mathfrak{h})$-linear combination of ordered monomials $Y_{1}^{\alpha_{1}} \cdots Y_{n}^{\alpha_{n}}$. An element of $A$ assigns to each such monomial a number $c_{\alpha}(a)$, and the map

$$
A \rightarrow K\left[\left[x_{1}, \ldots, x_{n}\right]\right], a \mapsto \sum_{\alpha \in N N^{n}} \frac{c_{\alpha}}{\alpha !} \mathbf{x}^{\alpha},
$$

where $\alpha !:=\prod_{i} \alpha_{i} !$, is the required algebra isomorphism. To see where the factor (1 divided by) $\alpha$ ! comes from, let $a_{\alpha}$ denote the unique element of $A$ that assigns 1 to $Y_{1}^{\alpha_{1}} \cdots Y_{n}^{\alpha_{n}}$ and 0 to all other ordered monomials in the $Y_{i}$. Then a straightforward computation shows that

$$
\left(a_{\alpha} \cdot a_{\beta}\right)\left(Y_{1}^{\alpha_{1}+\beta_{1}} \cdots Y_{n}^{\alpha_{n}+\beta_{n}}\right)=\left(\begin{array}{c}
\alpha+\beta \\
\alpha, \beta
\end{array}\right)
$$

while the value on all other ordered monomials is zero. This implies that

$$
a_{\alpha} \cdot a_{\beta}=\left(\begin{array}{c}
\alpha+\beta \\
\alpha, \beta
\end{array}\right) a_{\alpha+\beta},
$$

a set of identities (with varying $\alpha$ and $\beta$ ) shared by the "divided monomials" $\frac{\mathbf{x}^{\alpha}}{\alpha !}$. This explains the factorials appearing in the denominator, but it does more than that: in positive characteristic, divided power series are in many ways more natural than ordinary power series (and their algebra is no longer isomorphic to the algebra of formal power series). Blattner's construction automatically yields this algebra of divided power series. $^{2}$

\footnotetext{
2 It turns out that, in positive characteristic, the Lie algebra of derivations of the algebra of divided power series has finite-dimensional simple sub-algebras, and these Cartan-type Lie algebras are the main source of additional simple Lie algebras when passing from the classification in characteristic zero to the classification in positive characteristic [32, Chapters 2 and 4].
} 
Going back to characteristic zero, this explicit isomorphism between $A$ and the algebra of formal power series gives the following realisation formula.

Theorem 3.4 (Realisation Formula) Let $(\mathfrak{g}, \mathfrak{h})$ be a transitive pair. Let $Y_{1}, \ldots, Y_{n}$ be a basis of a vector space complement of $\mathfrak{h}$ in $\mathfrak{g}$. Then the map $\phi$ sending $X \in \mathfrak{g}$ to

$$
\phi(X)=\sum_{i=1}^{n}\left(\sum_{\alpha \in \mathbb{N}^{n}} \frac{a_{e_{i}}\left(Y_{1}^{\alpha_{1}} \cdots Y_{n}^{\alpha_{n}} X\right)}{\alpha !} \mathbf{x}^{\alpha}\right) \frac{\partial}{\partial x_{i}},
$$

where $e_{i}=(0, \ldots, 0,1,0, \ldots, 0)$ with the 1 on the ith position, is a realisation of $(\mathfrak{g}, \mathfrak{h})$.

So to compute the coefficient of $\mathbf{x}^{\alpha}$ in the coefficient of $\frac{\partial}{\partial x_{i}}$ in $\phi(X)$, one expresses $Y_{1}^{\alpha_{1}} \cdots Y_{n}^{\alpha_{n}} X$ as a $U(\mathfrak{h})$-linear combination of ordered monomials in the $Y_{j}$, and divides the (constant part of the) coefficient of $Y_{i}=Y_{1}^{0} \cdots Y_{i-1}^{0} Y_{i}^{1} Y_{i+1}^{0} \cdots Y_{n}^{0}$ by $\alpha$ !. Note that $X \in \mathfrak{g}$ is mapped to a derivation of non-negative order if and only if $a_{e_{i}}(X)$ is zero for all $i$, which in turn is equivalent to the statement that $X$ lies in $\mathfrak{h}$. So $\phi$ is, indeed, a realisation of $(\mathfrak{g}, \mathfrak{h})$. The fact that its kernel is the largest ideal of $\mathfrak{g}$ contained in $\mathfrak{h}$ is straightforward.

Example 3.5 Let $\mathfrak{g}=\mathfrak{s l}_{2}=\langle F, H, E\rangle$ with the usual commutation relations, let $\mathfrak{h}=\langle F, H\rangle$, and choose the complementary basis vector $Y_{1}:=E$. The linear function $a_{1}$ takes $E$ to 1 and all other ordered monomials $F^{i} H^{j} E^{k}$ to 0 . To compute $\phi(E)$, we have to consider powers $E^{d} E=E^{d+1}$, which under $a_{1}$ are mapped to 1 if $d=0$ and to 0 otherwise. Hence $\phi(E)=\frac{\partial}{\partial x}$. To compute $\phi(H)$, compute

$$
E^{d} H=E^{d-1} H E+E^{d-1}[E, H]=E^{d-1} H E-2 E^{d}=\cdots=H E^{d}-2 d E^{d} .
$$

Under $a_{1}$ this maps to 0 for $d=0$ or $d>1$ and to -2 for $d=1$. Hence $\phi(H)=$ $-2 x \frac{\partial}{\partial x}$. Finally, compute

$$
\begin{aligned}
E^{d} F & =E^{d-1} F E+E^{d-1} H=E^{d-1} F E+H E^{d-1}-2(d-1) E^{d-1} \\
& =\cdots=F E^{d}+d H E^{d-1}-d(d-1) E^{d-1}
\end{aligned}
$$

under $a_{1}$ this maps to 0 unless $d=2$, in which case it maps to -2 . Thus $\phi(F)=$ $\frac{-2}{2 !} x^{2} \frac{\partial}{\partial x}=-x^{2} \frac{\partial}{\partial x}$. Up to a sign, this is exactly the realisation found in Example 1.3.

So far we have sketched the proof of the existence of realisations. The uniqueness part of the Realisation Theorem follows from a universal property of the $U(\mathfrak{g})$-module $A$. For this, first note that $A$ comes equipped with a natural $U(\mathfrak{h})$-module map $a \mapsto a(1)$ into $K$ (which, in terms of formal power series, corresponds to evaluation at zero). Now given any other $U(\mathfrak{g})$-module $B$ together with a $U(\mathfrak{h})$-module map $\beta$ into $K$, there is a unique $U(\mathfrak{g})$-module map $\alpha: B \rightarrow A$ that makes the following diagram commute: 


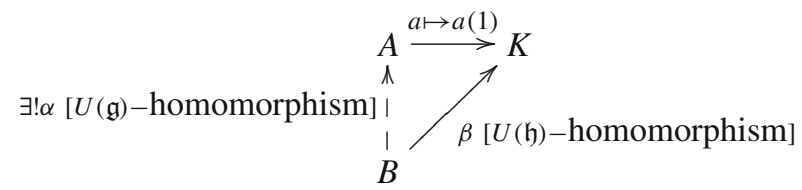

Indeed, $\alpha$ is forced to map $b$ to the element $u \mapsto \beta(u b)$ of $A$, and this map does the trick. ${ }^{3}$

Now a second realisation of $\mathfrak{g}$ gives rise, through the same fixed isomorphism of $A$ with the algebra of formal power series, to a second homomorphism $\phi_{2}: \mathfrak{g} \rightarrow \operatorname{Der}(A)$ in addition to the first homomorphism $\phi: \mathfrak{g} \rightarrow \operatorname{Der}(A)$ that we started with. Taking $B$ above equal to $A$ as a space, but with $U(\mathfrak{g})$-action coming from $\phi_{2}$, and taking $\beta$ equal to the map sending $b \in B$ to $b(1)$, the above shows that there is a unique $U(\mathfrak{g})$-module map $\alpha: B \rightarrow A$ such that $(\alpha b)(1)=b(1)$. This property implies, as above, that $\alpha b=u \mapsto(u b)(1)$. Somewhat lengthy, but straightforward computations show that $\alpha$ is an algebra isomorphism from $B=A$ to $A$ intertwining the realisations $\phi$ and $\phi_{2}$ as required by the theorem. This concludes our description of Blattner's proof from the book.

Remark 3.6 If $K$ equals $\mathbb{R}$ or $\mathbb{C}$, then one can show that the Realisation Formula yields a realisation consisting of vector fields with a positive radius of convergence around the origin [7]. Moreover, the unique formal coordinate change mapping that realisation to any other convergent realisation can be shown to be convergent, as well. Hence the Realisation Theorem implies that the classification of transitive Lie algebras of convergent vector fields in $n$ variables is the same as that of effective transitive pairs $(\mathfrak{g}, \mathfrak{h})$ where $\mathfrak{h}$ has codimension $n$ in $\mathfrak{g}$.

\section{Morozov and Dynkin: Primitive Lie Algebras}

While transitive Lie algebras are classified, up to coordinate changes, by effective transitive pairs, the classification of such pairs themselves in codimensions larger than two is very elaborate. Lie claims to have completed the case of codimension 3, but did not bother to publish the complete result (see [20]) — although he usually did not eschew lengthy computations in his books. Beyond codimension three, the classification of transitive Lie algebras may well remain out of reach.

But, as we saw in last section's discussion of Lie's classification in two variables, transitive pairs of codimension $m$ give rise to (in general, non-unique) sequences of primitive pairs whose codimensions add up to $m$. Thus it makes sense to try and classify at least these. This classification turns out to be very beautiful. Recall that a primitive pair is a transitive pair $(\mathfrak{g}, \mathfrak{h})$ with $\mathfrak{h}$ a maximal subalgebra in $\mathfrak{g}$. Adding the requirement that the pair be effective, i.e., that $\mathfrak{h}$ does not contain any non-zero $\mathfrak{g}$-ideal, turns out to leave only few possibilities for $\mathfrak{g}$. Here is the first theorem in that direction.

\footnotetext{
3 This construction is dual to the more familiar induction of representations from a smaller group to a larger group, and the fact that $U(\mathfrak{h})$-homomorphisms $B \rightarrow K$ are in one-to-one correspondence with $U(\mathfrak{g})$-homomorphisms $B \rightarrow A$ is the analogue of Frobenius reciprocity in this setting.
} 
Theorem 4.1 (Morozov [22]) Suppose that $(\mathfrak{g}, \mathfrak{h})$ is an effective primitive pair. Then either $\mathfrak{g}$ is simple, or else we are in one of the following two situations:

(1) $\mathfrak{g}$ is the direct sum $\mathfrak{k} \oplus \mathfrak{k}$ of two isomorphic simple Lie algebras and $\mathfrak{h}$ is the diagonal subalgebra consisting of all pairs $(X, X), X \in \mathfrak{k} ;$ or

(2) $\mathfrak{g}$ is the semi-direct product $\mathfrak{h} \ltimes \mathfrak{m}$ with $\mathfrak{m}$ an irreducible and faithful $\mathfrak{h}$-module equipped with trivial Lie bracket: $[\mathfrak{m}, \mathfrak{m}]=0$, and with $\mathfrak{h}$ is semisimple plus a center of dimension at most 1.

Conversely, in the latter two cases $(\mathfrak{g}, \mathfrak{h})$ is primitive and effective.

This theorem reduces the classification of effective primitive pairs to that of maximal subalgebras $\mathfrak{h}$ of simple Lie algebras $\mathfrak{g}$ (for which the pair $(\mathfrak{g}, \mathfrak{h})$ is trivially primitive). We will discuss that classification below, after sketching a proof of Morozov's result.

First assume that $\mathfrak{g}$ is not semisimple. A direct consequence of this is that $\mathfrak{g}$ has a non-zero Abelian ideal $\mathfrak{m}$. Then $\mathfrak{m}$ is not contained in $\mathfrak{h}$ by effectiveness, so $\mathfrak{h}+\mathfrak{m}$ is a subalgebra of $\mathfrak{g}$ strictly containing $\mathfrak{h}$. Hence by primitivity we have $\mathfrak{g}=\mathfrak{h}+\mathfrak{m}$ as vector spaces. But then $\mathfrak{h} \cap \mathfrak{m}$, being closed under taking brackets with $\mathfrak{h}$ and under taking under (zero) brackets with $\mathfrak{m}$, is an ideal in $\mathfrak{g}$, hence zero by effectiveness. Hence $\mathfrak{g}=\mathfrak{h} \oplus \mathfrak{m}=\mathfrak{h} \ltimes \mathfrak{m}$ as claimed. Any subspace $\mathfrak{i}$ of $\mathfrak{m}$ closed under taking brackets with $\mathfrak{h}$ leads to a subalgebra $\mathfrak{h} \ltimes \mathfrak{i}$ of $\mathfrak{g}$, hence $\mathfrak{i}$ equals zero or all of $\mathfrak{m}$; this proves that $\mathfrak{m}$ is an irreducible $\mathfrak{h}$-module. Finally, consider the kernel of the homomorphism $\mathfrak{h} \rightarrow \operatorname{End}(\mathfrak{m})$ sending $X$ to $[X,$.$] . This kernel is a \mathfrak{g}$-ideal, hence zero. We conclude that the representation of $\mathfrak{h}$ on $\mathfrak{m}$ is faithful, as required. The only Lie algebras $\mathfrak{h}$ with faithful, irreducible representations are those that are semisimple with a center of dimension at most one (which then acts by means of scalars on $\mathfrak{m}$ ).

Next assume that $\mathfrak{g}$ is semisimple, and write $\mathfrak{g}$ as the direct sum of a simple ideal $\mathfrak{k}$ and a semisimple ideal $\mathfrak{l}$, with $[\mathfrak{k}, \mathfrak{l}]=0$. Then $\mathfrak{k}, \mathfrak{l}$ are both not contained in $\mathfrak{h}$ by effectiveness, and hence $\mathfrak{g}=\mathfrak{h}+\mathfrak{k}=\mathfrak{h}+\mathfrak{l}$ by primitivity. Let $\pi_{\mathfrak{k}}$ denote the projection $\mathfrak{g} \rightarrow \mathfrak{k}$ with kernel $\mathfrak{l}$. Then the equality $\mathfrak{g}=\mathfrak{h}+\mathfrak{l}$ shows that the restriction of $\pi_{\mathfrak{k}}$ to $\mathfrak{h}$ is surjective onto $\mathfrak{k}$. On the other hand, the kernel of this restriction is $\mathfrak{h} \cap \mathfrak{l}$, which is an ideal in $\mathfrak{g}$ and hence zero by effectiveness. Hence $\pi_{\mathfrak{k}}$ restricts to an isomorphism $\mathfrak{h} \rightarrow \mathfrak{k}$. For entirely the same reason, the projection $\pi_{\mathfrak{l}}$ onto $\mathfrak{l}$ along $\mathfrak{k}$ restricts to an isomorphism $\mathfrak{h} \rightarrow \mathfrak{l}$. Hence $\mathfrak{k}$ and $\mathfrak{l}$ are isomorphic simple Lie algebras, and $\mathfrak{h}$ sits inside their direct sum as a diagonal subalgebra. This concludes the proof of the first part of Morozov's theorem. The converse, that the pairs under (1) and (2) are effective and primitive, is straightforward.

Example 4.2 Take $\mathfrak{h}=\mathfrak{g l}_{2}$ and $\mathfrak{m}$ equal to the standard two-dimensional module. Then the pair $(\mathfrak{h} \ltimes \mathfrak{m}, \mathfrak{h})$ corresponds to the primitive Lie algebra of Type (6) in Table 1.

As mentioned before, Morozov's theorem reduces the classification of (finitedimensional) effective, primitive pairs to that of maximal subalgebras of simple Lie algebras $\mathfrak{g}$. The following theorem deals with the case where the field is algebraically closed and $\mathfrak{g}$ is classical, that is, $\mathfrak{g}$ is isomorphic to the special linear algebra $\mathfrak{s l}_{n}$, the special orthogonal algebra $\mathfrak{s o}_{n}$, or the symplectic algebra $\mathfrak{s p}_{2 m}$. Let $V$ be the standard representation of $\mathfrak{g}$, that is, $V$ equals $K^{n}$ in the first two cases and $V=K^{2 m}$ in the last case. In the last two cases, $V$ is equipped with a bilinear form which is symmetric 
in the orthogonal case and skew-symmetric in the symplectic case. Now let $\mathfrak{h}$ be a maximal subalgebra of $\mathfrak{g}$. Then Dynkin classifies the possibilities as follows [8]:

$\mathfrak{h}$ acts reducibly on $V$ in this case, one of the following holds.

(1) $\mathfrak{h}$ is a maximal parabolic subalgebra of $\mathfrak{g}$. These are of the form

$$
\mathfrak{p}\left(V^{\prime}\right):=\left\{g \in \mathfrak{g} \mid g V^{\prime} \subseteq V^{\prime}\right\}
$$

with $V^{\prime}$ a proper subspace of $V$, totally isotropic in case $\mathfrak{g}$ is orthogonal or symplectic. Moreover, if $\mathfrak{g}=\mathfrak{o}_{2 m}$, then $\operatorname{dim} U \neq m-1$.

(2) $\mathfrak{g}=\mathfrak{o}(V)$, and $\mathfrak{h}=\mathfrak{o}(U) \oplus \mathfrak{o}\left(U^{\perp}\right)$ for some non-degenerate $U, 0 \subsetneq U \subsetneq V$.

(3) $\mathfrak{g}=\mathfrak{s p}(V)$, and $\mathfrak{h}=\mathfrak{s p}(U) \oplus \mathfrak{s p}\left(U^{\perp}\right)$ for some non-degenerate (and hence even-dimensional) $U, 0 \subsetneq U \subsetneq V$.

$\mathfrak{h}$ acts irreducibly on $V$ then there are two possibilities.

$\mathfrak{h}$ is not simple then one of the following holds.

(1) $\mathfrak{h}=\mathfrak{s l}(V)$, where $V \cong V_{1} \otimes V_{2}$, and $\mathfrak{h}=\mathfrak{s l}\left(V_{1}\right) \oplus \mathfrak{s l}\left(V_{2}\right)$. Here $V_{1}, V_{2}$ have dimensions $\geq 2$.

(2) $\mathfrak{g}=\mathfrak{s p}(V)$, where $V \cong V_{1} \otimes V_{2}$, and $\mathfrak{h}=\mathfrak{s p}\left(V_{1}\right) \oplus \mathfrak{o}\left(V_{2}\right)$. Here $\operatorname{dim} V_{1} \geq$ 2 , $\operatorname{dim} V_{2} \geq 3$ but either $\operatorname{dim} V_{2} \neq 4$ or $\left(\operatorname{dim} V_{1}\right.$, $\left.\operatorname{dim} V_{2}\right)$ equals $(2,4)$. Moreover, $V_{1}$ is equipped with a non-degenerate skew bilinear form, and $V_{2}$ with a non-degenerate symmetric bilinear form, such that the skew form on $V$ is the product of the two.

(3) $\mathfrak{g}=\mathfrak{o}(V)$, where $V \cong V_{1} \otimes V_{2}$, and $\mathfrak{h}=\mathfrak{o}\left(V_{1}\right) \oplus \mathfrak{o}\left(V_{2}\right)$. Here $\operatorname{dim} V_{1}, \operatorname{dim} V_{2} \geq 3$ but $\neq 4$. Moreover, each $V_{i}$ is equipped with a non-degenerate symmetric bilinear form, such that the symmetric form on $V$ is the product of the two.

(4) $\mathfrak{g}=\mathfrak{o}(V)$, where $V \cong V_{1} \otimes V_{2}$, and $\mathfrak{h}=\mathfrak{s p}\left(V_{1}\right) \oplus \mathfrak{s p}\left(V_{2}\right)$. Here $\operatorname{dim} V_{1}, \operatorname{dim} V_{2} \geq$ 2. Moreover, each $V_{i}$ is equipped with a non-degenerate skew bilinear form, such that the symmetric form on $V$ is the product of the two.

$\mathfrak{h}$ is simple then one of the following holds.

(1) $\mathfrak{g}=\mathfrak{s l}(V)$, and $\mathfrak{h}=\mathfrak{o}(V)$ for some non-degenerate symmetric bilinear form on $V$.

(2) $\mathfrak{g}=\mathfrak{s l}(V)$, and $\mathfrak{h}=\mathfrak{s p}(V)$ for some non-degenerate skew bilinear form on $V$.

(3) $\mathfrak{g}=\mathfrak{s l}(V)$, and $\mathfrak{h}$ leaves invariant no bilinear form on $V$.

(4) $\mathfrak{g}=\mathfrak{o}(V)$, and $\mathfrak{h}$ leaves invariant the symmetric bilinear form on $V$ defining $\mathfrak{g}$.

(5) $\mathfrak{g}=\mathfrak{s p}(V)$, and $\mathfrak{h}$ leaves invariant the skew bilinear form on $V$ defining $\mathfrak{g}$.

Conversely, still assuming that $\mathfrak{g}$ is classical simple, if $(\mathfrak{g}, \mathfrak{h})$ appears in the above list, then it is, as a rule, a primitive pair. Only in the last three cases, there are exceptions to this rule, and they are listed in Table 1 of [8].

This classification of maximal subalgebras of simple Lie algebras has been implemented in the programme Lie [5,18]. Dynkin has also classified the maximal subalgebras of the exceptional simple Lie algebras; see [9]. By an elegant result due to Karpelevich [17], one knows in advance that these are all parabolic or reductive. 
Wir halten uns mit diesen Rechnungen nicht weiter auf. Nur eins wollen wir erwähnen. Es zeigt sich nämlich, dass jede transitive Gruppe des Raumes $x, y, z$ auf eine solche Form gebracht werden kann, dass die Coefficienten von $p, q$ und $r$ ganze Functionen von $x, y, z$ und von gewissen Exponentialausdrücken: $e^{\lambda_{1}}, e^{\lambda_{2}}, \ldots$ werden, unter $\lambda_{1}, \lambda_{2} \ldots$ lineare Functionen von $x, y, \&$ verstanden*). Höchst wahrscheinlich ist, dasa ein ähnliches Gesetz auch für die transitiven Gruppen des Raumes ron $n$ Dimensionen gilt.

Fig. 1 Fragment from [21, p. 177]

\section{Realisations with Nice Coefficients}

This overview paper started off with Lie's problem of classifying transitive Lie algebras of vector fields in $n$ variables. Through the work of Guillemin-Sternberg and Blattner we have seen that this is equivalent to classifying effective transitive pairs of Lie algebras. Among these, the primitive pairs can actually be classified, as we have seen in the previous section.

Now we will complete the circle as follows: given an effective transitive pair $(\mathfrak{g}, \mathfrak{h})$, we know that a realisation in terms of formal power series exists, or even a realisation in terms of convergent power series for $K=\mathbb{R}$ or $\mathbb{C}$ - see Remark 3.6. But sometimes we can actually find realisations with nicer coefficients, such as polynomials, rational functions, or exponentials. Regarding such realisations, Lie expressed the opinion in Fig. 1, translated as follows.

It turns out, that every transitive group of 3-space with coordinates $x, y, z$ can be brought to a form in which the coefficients of $p, q$, and $r$ (Lie's notation for $\frac{\partial}{\partial x}, \frac{\partial}{\partial y}$, and $\left.\frac{\partial}{\partial z}-J . D.\right)$ are polynomial functions of $x, y, z$, and certain exponential expressions $e^{\lambda_{1}}, e^{\lambda_{2}}, \ldots$, where $\lambda_{1}, \lambda_{2}, \ldots$ denote linear functions of $x, y, z$. Very probably a similar statement holds for the transitive groups of $n$-space.

In fact, such realisations always exist when the pair is very imprimitive in the sense of the following theorem.

Theorem 5.1 (Draisma [7]) Let $(\mathfrak{g}, \mathfrak{h})$ be an effective transitive pair of codimension $n$, and suppose that there exists a chain

$$
\mathfrak{g}=\mathfrak{g}_{n} \supseteq \mathfrak{g}_{n-1} \supseteq \cdots \supseteq \mathfrak{g}_{1} \supseteq \mathfrak{g}_{0}=\mathfrak{h}
$$

of subalgebras $\mathfrak{g}_{i}$ with codimension $n-i$ for $i=0, \ldots, n$. Then $(\mathfrak{g}, \mathfrak{h})$ has a realisation $\phi: X \mapsto \sum_{i=1}^{n} f_{i, X} \frac{\partial}{\partial x_{i}}$ where for all $X$ and $i$ the coefficient $f_{i, X}$ is a polynomial in the $x_{i}$ and (finitely many) expressions $\exp \left(\lambda x_{i}\right)$ with coefficients $\lambda$ in the algebraic closure of $K$.

The proof of this theorem uses the Realisation Formula of Theorem 3.4: as an ordered basis of a complementary subspace to $\mathfrak{h}$ one takes $Y_{i} \in \mathfrak{g}_{i} \backslash \mathfrak{g}_{i-1}$ for $i=$ $1, \ldots, n$, and then the coefficients $f_{i, X}$ can be shown to satisfy non-trivial linear 
ordinary differential equations in all of the variables. For details see [7]. There are variants of this theorem that yield polynomial realisations, such as the following.

Theorem 5.2 (Draisma [7]) Let $(\mathfrak{g}, \mathfrak{h})$ be an effective transitive pair of codimension $n$, and suppose that $\mathfrak{g}$ has subalgebra $\mathfrak{m}$ complementary to $\mathfrak{h}$ such that for each element $X \in \mathfrak{m}$ the linear map $\operatorname{ad}(X): \mathfrak{g} \rightarrow \mathfrak{g}, Y \mapsto[X, Y]$ is nilpotent. Then $(\mathfrak{g}, \mathfrak{h})$ has a realisation in which all coefficients are polynomial.

In this case, the Realisation Formula with $Y_{1}, \ldots, Y_{n}$ any ordered basis of $\mathfrak{m}$ gives such a polynomial parameterisation.

Example 5.3 In Morozov's Theorem 4.1 the second type of primitive pairs are of the form described in this theorem. Indeed, assume that $\mathfrak{g}=\mathfrak{h} \ltimes \mathfrak{m}$ with $\mathfrak{m}$ an Abelian ideal, and choose a basis $Y_{1}, \ldots, Y_{m}$. Then we have $Y_{i} Y_{j}=Y_{j} Y_{i}$ in $U(\mathfrak{g})$, so that the linear part of $Y_{1}^{\alpha_{1}} \cdots Y_{n}^{\alpha_{n}} Y_{i}$ is only non-zero when all $\alpha_{j}$ are zero. This shows that $Y_{i}$ is mapped to $\frac{\partial}{\partial x_{i}}$ in the realisation formula. On the other hand, for $X \in \mathfrak{h}$ we have

$$
Y_{j} X=X Y_{j}+\sum_{i=1}^{n} a_{i j} Y_{i}
$$

where $\left(a_{i j}\right)_{i j}$ is the matrix of $-\operatorname{ad}_{\mathfrak{m}} X$ with respect to the basis $Y_{1}, \ldots, Y_{n}$. The linear part of a higher-degree monomial in the $Y_{i}$ times $X$ is again zero. Hence we find that the realisation maps $X$ to $\sum_{j}\left(\sum_{i} a_{i j} x_{j} \frac{\partial}{\partial x_{i}}\right)$.

Example 5.4 If $\mathfrak{g}$ is a complex semisimple Lie algebra and $\mathfrak{p}$ is a parabolic subalgebra, then $\mathfrak{p}$ has a complement as in this theorem, and as a result the pair $(\mathfrak{g}, \mathfrak{p})$ has a realisation with polynomial coefficients. For explicit formulas, see [27].

Remark 5.5 Table 2 was compiled as follows: first, I re-did the classification of effective, transitive pairs using only purely Lie-algebraic arguments. It turns out that the primitive pairs are of the form in Theorem 5.2, while the non-primitive pairs are trivially of the form in Theorem 5.1. I then used the pairs, with the appropriate basis of a complement, as input to a GAP implementation of the Realisation Formula $[4,10]$, truncating the power series at high enough degree to recognise the relevant polynomials and exponentials (this, too, can be done automatically, because the proofs of both theorems above gives explicit information on the degrees of the polynomials and on which exponentials will appear).

As remarked earlier, Lie's Gruppenregister [20] does not contain a complete list of transitive Lie algebras in three variables. Indeed, the ones that are missing there are precisely the very imprimitive ones, for which Theorem 5.1 ensures the existence of a nice realisation. This fact, together with Lie's classification of the other pairs, confirms Lie's quote above in the case of three variables.

There are pairs $(\mathfrak{g}, \mathfrak{h})$ for which a realisation with polynomial coefficients exists that can probably not be obtained directly from the Realisation Formula.

Example 5.6 In Morozov's Theorem 4.1, the first class of primitive pairs $(\mathfrak{k} \oplus \mathfrak{k}, \mathfrak{h})$ (with $\mathfrak{h}$ the diagonal subalgebra) have polynomial realisations. A proof of this fact 
uses the simply connected algebraic group $P$ with Lie algebra k: let $H$ be the diagonal subgroup of $P \times P$. Then the action of $P \times P$ by left multiplication on the quotient $(P \times P) / H$ differentiates to a realisation of $\mathfrak{p} \times \mathfrak{p}$ by vector fields on this quotient. More precisely, for every open affine subset $U$ of the base point $(e, e) H$ (with stabiliser $H$ ) we obtain a Lie algebra homomorphism $\mathfrak{p} \times \mathfrak{p} \rightarrow \operatorname{Der} K[U]$ such that $\mathfrak{h}$ is the preimage of all vector fields in $K[U]$ vanishing at the base point. If we can choose $U$ to be isomorphic to an affine space (and not just to some affine variety), then $K[U]$ is a polynomial ring and we have the desired polynomial realisation. By the Bruhat decomposition, $P$ contains an open neighbourhood $V$ of $e$ that is isomorphic to an affine space, and we may take $U$ to be the image of $V \times\{e\}$ in the quotient $P \times P / H$. This construction generalises, in fact, to pairs corresponding to spherical varieties $[2,3]$.

Although I have no proof, I think that in the preceding class of examples no choice of a basis complementary to $\mathfrak{h}$ leads the Realisation Formula to output a polynomial realisation. Observe that this is not in contradiction with the uniqueness part in the Realisation Theorem: although any realisation of a pair $(\mathfrak{g}, \mathfrak{h})$ is related to a fixed realisation obtained from the Realisation Formula by a unique formal coordinate change, this coordinate change, which depends on countably many independent coefficients in the formal power series images of $x_{1}, \ldots, x_{n}$, will typically not come from a change of (basis of a) complement to $\mathfrak{h}$, which is determined by finitely many independent coefficients, and which is all the data that goes into the Realisation Formula. This circumstance and Lie's quote above lead to two interesting research problems:

- First, might Lie's observation literally hold true in higher dimensions? In other words, does every transitive pair have a realisation with coefficients that are polynomials in the variables $x_{i}$ and simple exponentials $\exp \left(\lambda x_{i}\right)$ ? Might such a realisation always be obtainable with the Realisation Formula? Presently I am inclined to answer both questions in the negative, but I have no counter-examples yet. For instance, when allowing exponentials, the Realisation Formula does yield allowed realisations for the "diagonal pairs" in the previous example [7]. My search for actual counter-examples would start at primitive pairs $(\mathfrak{g}, \mathfrak{h})$ from Dynkin's list with $\mathfrak{g}$ classical and its standard module an irreducible $\mathfrak{h}$-module.

- Taking Lie's observation less literally, one might change the condition

(1) that the coefficients of the vector fields be solutions to linear o.d.e.s with constant coefficients in each of the variables to the condition

(2) that the Lie algebra as a whole be the solution of a system of linear p.d.e.s (not necessarily with constant coefficients).

For example, the Lie algebra of type (8) in Table 1 is the solution to the determining system (2), but in condition (2) we do not insist that the system have constant coefficients.

This condition (2) was suggested by Mohamed Barakat at the 2010 Oberwolfach mini-workshop Algebraic and analytic techniques for polynomial vector fields. There is a beautiful challenge here: start with a transitive pairs $(\mathfrak{g}, \mathfrak{h})$ and construct, preferably in the coordinate-free manner introduced by Blattner, a system of linear p.d.e.s to which (a realisation of) the pair is the solution. Then find necessary and sufficient 
conditions for the system to have "constant coefficients"-which would correspond to the class described by Lie.

Especially the second research question seems very promising to me, and with it I conclude this overview paper on Lie algebras of vector fields.

Open Access This article is distributed under the terms of the Creative Commons Attribution Noncommercial License which permits any noncommercial use, distribution, and reproduction in any medium, provided the original author(s) and source are credited.

\section{References}

1. Blattner, R.J.: Induced and produced representations of Lie algebras. Trans. Am. Math. Soc. 144, 457474 (1969)

2. Brion, M.: Variétés sphériques. Notes of the S.M.F. session Opérations Hamiltoniennes et opérations de groupes algébriques. Grenoble 1997. http://www-fourier.ujf-grenoble.fr/ mbrion/notes.html

3. Brion, M., Luna, D., Vust, T.: Espaces homogènes sphériques. Invent. Math. 84, 617-632 (1986)

4. Draisma, J.: blattner, an implementation of the realisation formula. GAP-code: http://www.win. tue.nl/ jdraisma/index.php?location=programs

5. Draisma, J.: maximal_subalgebras, an implementation of Dynkin's classification. LiE-code: http://www.win.tue.nl/ jdraisma/index.php?location=programs

6. Draisma, J.: Recognizing the symmetry type of o.d.e.'s. J. Pure Appl. Algebra 164(1-2), 109-128 (2001)

7. Draisma, J.: On a conjecture of Sophus Lie. In: Differential Equations and the Stokes Phenomenon. Proceedings of a Workshop held at Groningen University from May 28-30, 2001. World Scientific, Singapore (2002)

8. Dynkin, E.B.: Maximal subgroups of the classical groups. Am. Math. Soc. Transl. II. Ser. 6, 245-378 (1957)

9. Dynkin, E.B.: Semisimple subalgebras of semisimple Lie algebras. Am. Math. Soc. Transl. II. Ser. 6, 111-244 (1957)

10. The GAP Group.: GAP_Groups, Algorithms, and Programming, Version 4.3. http://www.gap-system. org (2002)

11. González-López, A., Kamran, N., Olver, P.J.: Lie algebras of first order differential operators in two complex variables. Am. J. Math. 114(6), 1163-1185 (1992)

12. González-López, A., Kamran, N., Olver, P.J.: Lie algebras of vector fields in the real plane. Proc. Lond. Math. Soc. III. Ser. 64(2), 339-368 (1992)

13. Guillemin, V.W.: Infinite dimensional primitive Lie algebras. J. Differ. Geom. 4, 257-282 (1970)

14. Guillemin, V.W., Sternberg, S.: An algebraic model of transitive differential geometry. Bull. Am. Math. Soc. 70, 16-47 (1964)

15. Helgason, S.: Differential geometry and symmetric spaces. In: Pure and Applied Mathematics, vol. XII. Academic Press, New York-London (1962)

16. Jacobson, N.: Lie Algebras. Dover, New York (1969, reprint)

17. Karpelevich, F.I.: Über nicht-halbeinfache maximale Teilalgebren halbeinfacher Liescher Algebren. Dokl. Akad. Nauk SSSR 76, 775-778 (1951)

18. van Leeuwen, M.A.A., Cohen, A.M., Lisser, B.: LiE: A Package for Lie Group Computations. Amsterdam (1992). http://wwwmathlabo.univ-poitiers.fr/ maavl/LiE/

19. Lie, S.: Vorlesungen über Differentialgleichungen mit bekannten infinitesimalen Transformationen. Bearbeitet und herausgegeben von G. Scheffers. B.G. Teubner, Leipzig (1891)

20. Lie, S.: Gruppenregister. Gesammelte Abhandlungen, vol. 5, pp. 767-773. B.G. Teubner, Leipzig (1924)

21. Lie, S., Engel, F.: Transformationsgruppen, vol. 3. B.G. Teubner, Leipzig (1893)

22. Morozov, V.V.: Sur les groupes primitifs. Rec. Math. Moscou N. Ser. 5, 355-390 (1939)

23. Olver, P.J.: Applications of Lie Groups to Differential Equations, 2nd edn. Graduate Texts in Mathematics, vol. 107. Springer, New York (1986)

24. Oudshoorn, W.R.,van der Put, M.: Lie symmetries and differential Galois groups of linear equations. Math. Comput. 71(237), 349-361 (2002) 
25. Reid, G.J.: Algorithms for reducing a system of pdes to standard form, determining the dimension of its solution space and calculating its Taylor series solution. J. Appl. Math. 2(4), 293-318 (1991)

26. Reid, G.J.: Finding abstract Lie symmetry algebras of differential equations without integrating determining equations. Eur. J. Appl. Math. 2(4), 319-340 (1991)

27. Richter, D.A.: $\mathbb{Z}$-gradations of Lie algebras and infinitesimal generators. J. Lie Theory $\mathbf{9}(1), 113-123$ (1999)

28. Robertz, D.: Janet bases and applications. In: Rosenkranz, M., et al. (eds.) Gröbner Bases in Symbolic Analysis. Radon Series on Computational and Applied Mathematics, vol. 2, pp. 139-168. Walter de Gruyter, Berlin (2007). Based on talks delivered at the special semester on Gröbner bases and related methods, Linz, Austria, May 2006

29. Schwarz, F.: Janet bases for symmetry groups. In: Buchberger, B., Winkler, F. (eds.) Gröbner Bases and Applications, pp. 221-234. Cambridge University Press (1998)

30. Sharpe, R.W.: Differential geometry: Cartan's generalization of Klein's Erlangen program. Graduate Texts in Mathematics, vol. 166. Springer, Berlin (1997)

31. Singer, I.M., Sternberg, S.: The infinite groups of Lie and Cartan. I: The transitive groups. J. Anal. Math. 15, 1-114 (1965)

32. Strade, H.: Simple Lie algebras over fields of positive characteristic. I: Structure theory. de Gruyter Expositions in Mathematics, vol. 38. de Gruyter, Berlin (2004) 